\title{
An Intelligent Electricity Meter RFID Receiving System Based on the Internet of Things Cloud Platform
}

\author{
Silei Shen ${ }^{1}$ and Chao Wang ${ }^{2 *}$ \\ ${ }^{1}$ Beijing Qunling Technology Energy Co., Ltd., China \\ ${ }^{2}$ Beijing University of Technology, Beijing, China \\ ${ }^{*}$ Corresponding author
}

\begin{abstract}
As an important part of intelligent power Grid, cloud computing is becoming more and more important in the development of intelligent grid with the development of the internet of things cloud platform. This paper introduces the application of cloud computing technology in the intelligent power grid, the working principle of RFID and the hardware design of the meter. The design takes MSP430 low power processor and ADE7848 chip as the core. In order to ensure the validity of the data, the design program reads data from ADE7848 to the memory every $100 \mathrm{~ms}$. Design of intelligent meter-reading system based on internet-connected cloud platform based on RFID technology. According to this system, users can observe the electricity usage in real time.
\end{abstract} RFID

Keywords_intelligent electricity meter; MSP430; ADE7848;

\section{The CONSTRUCTION OF THE INTERNET OF THINGS CLOUD PLATFORM}

According to the general definition, the Internet of Things refers to information sensing devices such as radio frequency identification (RFID), infrared sensors, global positioning systems, and laser scanners. Any item is connected to the Internet by agreement to exchange and communication aiming to reply a network to achieve intelligently identifies, locates, tracks, monitors, and manages as an important localized central information processing center between the wireless sensor network and the Internet the IoT cloud platform must have the following functions. (1) Business acceptance, opening and billing. (2) Information acquisition, storage, calculation, and display (3) Flexible application development in industry.

\section{Cloud Computing Technology ApPly IN INTELLIGENT GRID}

With the development of society, the type and quantity of grid data information together with service requests for users are rapid development the massive data and related processing requirements make the current grid information system unsatisfactory. In addition, the data distribution scattered in the intelligent grid, and the data types and related processing frequencies are not exactly same Therefore, while ensuring the intelligent grid has enough space for data storage, it also needs to have superior computing capabilities. Cloud computing stores data in a distributed manner. In addition, the reliability of cloud computing data is also maintained by its redundant storage and highly reliable stable software. Therefore, a intelligent grid cloud formed by the intelligent grid system of cloud computing architecture serves users and power systems in a transparent manner. It provides superior computing capabilities based on virtualization, storage, management of massive data, real-time scheduling and monitoring, to ensure that users and circuit systems are satisfied with their computing and data storage services.

The intelligent grid cloud is divided into four parts. The first part is the physical storage, which is the bottom of physical equipment facility in the cloud scope; the second part is the basic management , through the computing clusters and distributed systems it guarantees All storage devices in the inside of the intelligent grid cloud work together, save, manage, and secure data. The third part is the application interface, it reserved software interface to ensure that the corresponding application can be developed according to the required function or service at any time, and that it can be interfaced with the interface quickly and guarantee the stable expansion of the intelligent grid cloud; the fourth part is the advanced access, which provides desktop-like system operation and reduces the difficulty of operation.

Currently, there are mainly three types of electricity users: residential users, commercial users, and industrial users. Basely intelligent meters will be installed in each household. In addition, intelligent meters are also required in the each area, which constitutes the home and area networks. The electrical data information is transmitted to the cloud, and then it is processed by the algorithm preset in the intelligent grid cloud, so that optimal scheduling can be performed to ensure that no occurs overload or no-load operation. China's intelligent grid is still in its development stage, which means that it will develop many application services in the future. It can be developed by third-party software developers in accordance with the ports, so that it is easy to implement multiple services. Considering the cloud computing characteristics of the IoT operating platform, it prefer to introduce cloud computing technologies to build an IoT operating platform. The Internet of Things platform based on the CloudX 5 cloud platform.

Through the import of physical resource virtualization technology, applications in different industries and resources (storage, CPU, etc.) of different customers in the same industry can be shared, which operating on the Internet of Things 
operating platform For example, instead of allocating a fixed storage space for each customer, the customers used share virtual storage pool that spans physical storage devices.

Provides elastic scaling of resource requirements, such as sharing computing resources between different industry data intelligence analysis processing processes, or dynamically allocating storage resources from virtual storage pools when individual customer storage resources are exhausted, in order to satisfy customers as closely as possible with minimal resources Demand, and reduce operating costs while improving service quality.

Importing server clustering technology to associate a group of servers so that they appear to be the same server in many aspects of the outside world, thereby improving the overall performance and availability of the IoT operating platform.

It realizes the configuration and control of network nodes, the collection and calculation of information, and it can adopt distributed storage and distributed computing technology to realize the analysis and processing of massive data to meet the large amount of data and real-time data processing requirements.

\section{RFID SYSTEM WORKING PRINCIPLE}

\section{A. Hardware}

1) Tag. Composed of a coupling elements and chips, each tag has unique electronic data and attached to the object to identify the target.

2) Reader. Devices that read electronic tag information can be designed to be hand-held or stationary. It is used to transmit radio frequency signals and receive the signals reflected by the electronic tags. After processing, the tag data information is obtained.

3) Antenna. Pass device signals between electronic tags and readers to control data acquisition link to communication. Generally antennas and readers are integrated.

\section{B. Working Principle}

The working principle of RFID is the reader emits electromagnetic waves in a certain area. There is a resonant circuit inside the electronic tag. When the tag enters the magnetic field, it can generate inductive current to obtain energy, clock and instructions, and transmit the useful data in back-scatter modulation. The reader receives the data of this tag, decodes it, and sends it to the central information system for data processing. In this way, the reader can achieve contactless reading and identification of the data stored in the electronic tag through the antenna, aiming at automatically identifying the object.

\section{HARDWARE DESIGN OF AMMETER}

The whole hardware principle of the system mainly includes MSP430 low-power processor, integrated metering module, IC card interface, network interface module, LED display module, keyboard module, storage module, power supply module and so on.

\section{A. MSP430 Microcontroller}

The new intelligent meter requires real-time sampling of the input power signal and data processing of the sampled signal through an efficient decoding algorithm. This process is complex so it requires a fast and efficient CPU digital signal processing capability. On the one hand, for the sake of cost control. We used the MSP430 ferro-electric storage processor of TI Company. The processor has separate accumulators and multipliers. It has a 16-bit hardware timer and 6 independent programmable DMA channels (direct memory access channels) that can be used without affecting the speed and efficiency of the CPU. Transfer data between internal memory, on-chip peripherals, and external memory. While using ferro-electric memory to store and read data at the RAM operating speed greatly improves the speed. In the standby state, its operating current consumption is only $1 \mathrm{uA}$, meeting the requirements of energy saving and environmental protection.

\section{B. ADE7848 Metering IC}

The ADE7848 is a highly integrated measurement IC from Analog Devices. The measurement chip can read data such as active power, reactive power, voltage, and current. The ADE7858 uses a 3.3V supply to communicate with the MCU via SPI or C. To ensure the validity of the data, the design program reads the data from the ADE7848 to the memory every $100 \mathrm{~ms}$.

\section{RFID Radio Frequency Module}

At present, they widely used RFID cards on the domestic market generally using Mifare chips, and the device adopts the ISO/IEC 14443A protocol produced by Philips and the 13.56 $\mathrm{MHz}$ non-contact RC500 reader/writer chip. The following are the basic parameters of the RC500 chip.

Energy transmission - the principle of transformer, electronic tags passivity;

Working frequency -13.56 MHz;

Communication structure-half duplex;

Data rate $-105.9 \mathrm{kHz}$;

Interface type-parallel.

The MFRC500 chip supports all layers of the ISO 14443A protocol, and its analog circuitry consists of a RF transmitter module and a receiver module. The transmitter module can directly drive an antenna with a working distance of up to 10 $\mathrm{cm}$ without adding an active circuit. The receiver module can conveniently and quickly demodulate and decode card signals conforming to the ISO 14443A protocol.

The MFRC500 chip contains an 8-bit parallel micro-controller interface that automatically detects the type of interface to which it is connected. It also includes a configurable interrupt output and a bidirectional FIFO buffer. The MFRC500 chip contains a wide range of parallel interfaces that can be directly connected to a variety of 8-bit micro-processors, allowing greater flexibility and volatility when designing readers/terminals. For the data processing part of the received signal, the device focuses on the encapsulation and error detection of the ISO14443A frame (the monitoring 
method supports RC and parity check).he flexibility of the MFRC500 is also reflected in the fact that the chip can be autonomously configured through the state and control sections, adapting to different environments and maximizing the performance of the chip to achieve the most ideal state.

The RFID radio reader module mainly relies on the communication between the antenna and the electronic tag. Under the premise that the register has been preset, the MFRC500 chip will accurately modulate the transmission data according to the parameters to obtain a transmission signal, and through the drive of antenna , the signal is sent out in the form of electromagnetic wave as13.56 MHz. The RFID card in its RF range responds by using the load modulation and generates a response signal. After the response signal is received by the antenna, it sent to the signal receiving pin of the MFRC500 chip through the antenna matching circuit. The receiver module demodulates and decodes the received signal, processes the signal data according to the preset register parameters, and finally sends the data to the parallel interface of the chip to be read by the micro-controller.

\section{Wired Network Port Design}

RTL8019AS is a universal Ethernet interface chip manufactured by Raychem. The MCU communicates with the RTL8019AS via a 16-bit bus. Since the operating voltage of the RTL8019AS chip is $5 \mathrm{~V}$, and the $\mathrm{I} / \mathrm{O}$ interface voltage of the $\mathrm{MCU}$ is $3.3 \mathrm{~V}$, in order to protect the circuit, a protection resistor needs to be connected in series with the bus. While the RTL8019AS operates in jumper mode, the IO address is determined by IOS3-IOS0. The assay uses all floating, so IOS3-IOS0 is 0 , and the IO address is $300 \mathrm{H}$. Then select the 8-bit data mode. The A5 port is connected to the MCU memory chip select CS. When A8 is high and A5 is low, the MCU selects the RTL8019AS.

\section{SOFTWARE DESIGN PART}

\section{A. Main Program Flow}

First, in the main program, initialize each module of the system. Secondly, read the parameters of the measurement chip, and compare whether the current power consumption exceeds the maximum power allowed or other faults, and then turn off the power after a fault occurs, and start the fault alarm and wait for reset. Deal with. If it is not exceeded, the operating parameters are displayed by keyboard control while the standby mode enters low power mode. When an electronic tag is identified, part of the RFID module program is automatically run, and user-related data is displayed on the LED.

\section{B. RFID Identification Subroutine Design}

When the radio frequency IC card has read and write requirements, the system will trigger access to radio frequency IC card read and write subroutines. After the program enters, the card will be authenticated first. After the authentication is successful, the system MCU will add the electricity charge stored by the user in the card to the remaining electricity charge in the EEPROM. The result of the addition will be stored in the EEPROM and the IC card will be stored. The electricity value in the bill is cleared.

\section{Design of Metering Interruption Subroutines}

The energy metering subroutine runs in three steps. The first is to determine the billing period for the current value, the second is the accumulation of energy for the corresponding period, and the third is the deduction of the corresponding electricity bill. At this point, the accumulated power and the deducted electricity charge are the number of electrical energy represented by a pulse and the electricity charge. In addition, the operating voltage, current and other parameters can be directly read into the corresponding memory.

\section{CONCLUSION}

Through the above system design, users can use the electronic label to easily understand the power situation. The electronic label can be easily attached to a wallet or a mobile phone. On the other hand, intelligent meters use two-way power measurement methods to effectively prevent theft. Intelligent meters based on the Internet of Things cloud platform can achieve remote intelligent control. The power supply company can switch on and off specific power supply lines at any time, and can also set specific time-phased billing plans to save time and effort and have broad application prospects.

\section{REFERENCES}

[1] Zhang Wei, Shen Chen, Lu Qiang. Framework of The Power Grid System Part One Development of Power Network Monitoring from Centralized to Distribute Processing [J].Automation of Electric Power Systems.2004.28

[2] Xiao Shi Jie. Fully Promote the Power Grid Revolution to Drive Economic Innovation and Transformation [EB/OL] 2009. 2

[3] Zhang Bin, Sun Shi Yin. Cloud Computing and Smart Substation Distributed Computing [J].Information system engineering .2010:141-488

[4] Chen Xiao Chao. Study on Application of Cloud Computation in Technical Support System of Smart Grid Dispatching [J].East China Electric Power.2010.6

[5] Sun Jing.The Machine Communication Technology of Smart Grid [J].power system communications.2010.32

[6] Xu Qiang, Guo Ming, Xie Yong, Wang Hongwei. Design and Implementation of Real Time Even Handling of RFID [J] Logistics Technology.2007-01. 